\title{
BER Evaluation of Post-Meter PLC Services in CENELEC-C Band
}

\author{
Sadaf Moaveninejad*, Atul Kumar*, Davide Scazzoli*, Alessandro Piti*†, \\ Maurizio Magarini*, Stefano Bregni*, Giacomo Verticale* \\ ${ }^{*}$ Dipartimento di Elettronica, Informazione e Bioingegneria, Politecnico di Milano, Milano, Italy \\ Email: name.surname@ polimi.it \\ ${ }^{\dagger}$ Italian Regulatory Authority for Electricity Gas and Water (AEEGSI), Milano, Italy
}

\begin{abstract}
Low voltage, in-home power-line communications (PLC) networks allow direct communication between smart meters (SM) and in-home devices (IHD). In order to minimize security issues, in many deployment scenarios transmission takes place only towards the IHD to display consumption data, with no backwards channel. As a result, channel estimation is difficult and it is necessary to use robust transmission techniques to mitigate the effect of the impulsive noise within the PLC channel. Performance of such system must be evaluated by taking into account realistic interference and channel models for a broad range of configurations. In this work we focus on performance in terms of bit error rate (BER) of a narrowband PLC (NB-PLC) operating in the CENELEC-C band (125-140 kHz) taking into account realistic noise models. Our system is based on binary phase shift keying (BPSK) and quadrature phase shift keying (QPSK) modulation.
\end{abstract}

Keywords-Post-meter, PLC-C, NBPLC, Smart Home, Smart Grids, In Home Device, Smart Meter, CENELEC-C

\section{INTRODUCTION}

Powerline communications (PLC) has gained a lot of attention in the last two decades, especially in the context of smart grids. In PLC, information is transmitted on the same wire used for electric power transmission. So, there is no the need for new infrastructure with additional cost [1]. Smart meters (SM) are part of smart grid and provide information regarding power consumption, supply and demand [2]. However, in order to use this information, we need to establish a highly reliable communication [3] both in the link between SM and the data concentrator and in that between SM and in-home device (IHD). The so called in-home device is an example of postmeter service used in smart homes.

Regarding the communications link for monitoring flow of energy of in-home devices recently narrowband PLC (NBPLC) has been receiving popularity. NB-PLC works at low frequencies between $3-500 \mathrm{kHz}$ allowing low data rate which is suitable for energy consumption profiling. Moreover, NBPLC can cover longer distances and cheap modems can be deployed.

In Europe, the regulated bandwidth for NB-PLC is defined by the Comit Europen de Normalization Electrotechnique (CENELEC) signaling standard. This standard divides frequencies in the range $3-148.5 \mathrm{kHz}$ into four sub-bands. The
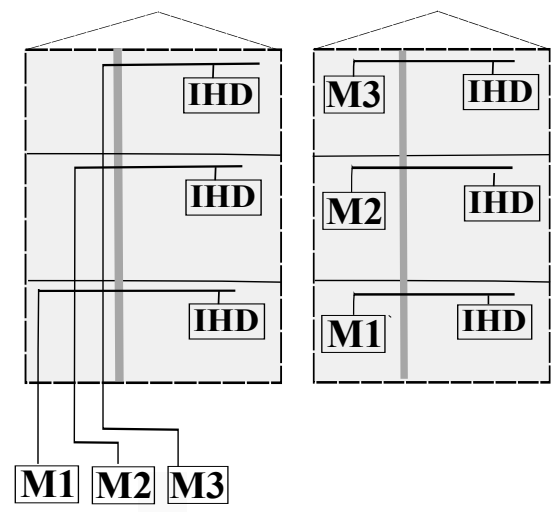

Fig. 1. Architecture of the link between Meters and IHDs in a residedential building

last three bands known as $\mathrm{B}, \mathrm{C}$, and $\mathrm{D}$ are reserved for the energy customers. The so called CENELEC-C band refers to frequency in range between $125-140 \mathrm{kHz}$ reserved for home network systems and developed by KNX international standard for home and building control [4]. With the advancements in concepts of smart grid and smart home, CENELEC-C band could be a suitable choice for home network systems and enable direct transmission of metering data from the SM to devices located near the customers premises [5]. Authors of [6] considered interoperability between different standards and protocols as a main issue in the rising market of smart home. In Table I, different NB-PLC technologies developed for home automation applications in the CENELEC-C band are reported [7] .

In this paper, we consider a system that communicates only in one-way from SM to IHD in order to display consumption information and enable real-time information services. Consequently, neither any commands and control signals nor channel information are sent back from user to the SM. The main advantage of such system is time reduction in tele-management and real-time access to the meter's data. However, no feedback is available in this one-way transmission. This causes some issues in channel estimation and signal detection at the receiver. Hence, robust transmission techniques are required 
TABLE I

STANDARDS AND TECHNICAL SPECIFICATIONS OF NB-PLC SUPPORTING CENELEC-C BAND [7]

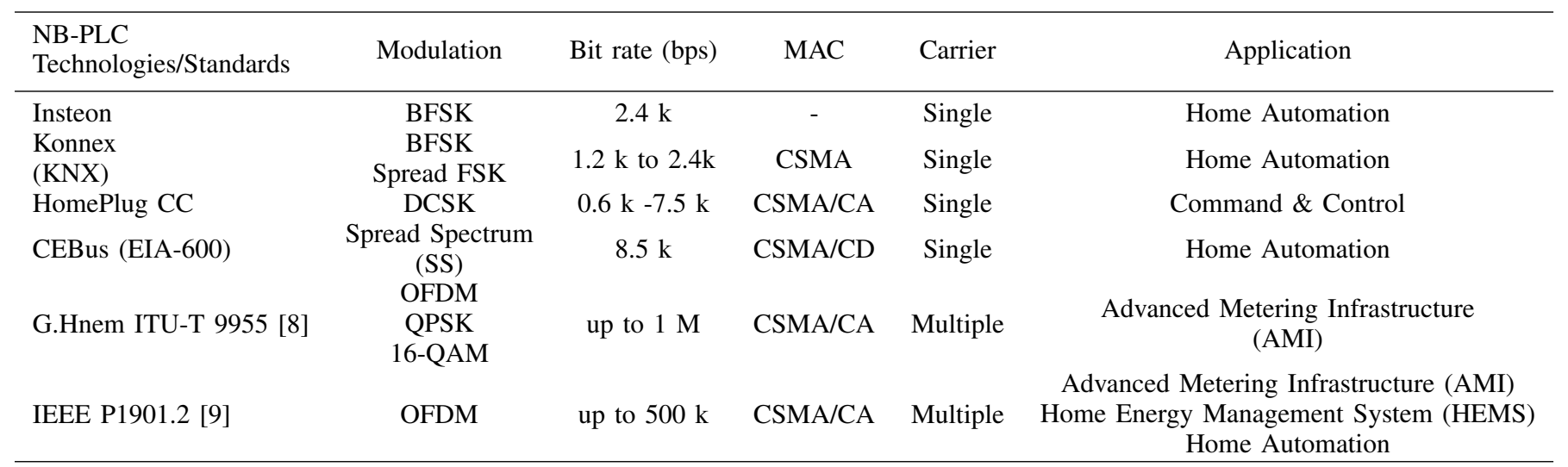

for such one-way communication.

There are two different ways to install meters in residential buildings which are shown in Fig. 1. In the first approach, the meters of all apartments are grouped together in one place in basement of the building and then from each meter there is a powerline to the corresponding apartment. Communication with meters in an underground location provides another challenge. PLCs provide a viable communication solution with these meters [10].

In the other configuration, each meter is installed inside each apartment. In both cases, the data of each individual household is collected by the SM and transmitted to the IHD through wireless or PLC links. The difference is the length of the channel between transmitter and receiver.

In this paper we focus on the first configuration to compare BER for different lengths of the links between SM and IHD and different number of multipath. These links are between SMs in basement and IHDs in first floor and third floor. For the first floor and third floor we defined the maximum length of the cable equal to $40 \mathrm{~m}$ and $80 \mathrm{~m}$ respectively.

The existing electrical wiring and power outlets may be considered as a link for data communication within the home. Design of PLC systems should take the noise, attenuation and multipath characteristic of communication into consideration. Consequently, suitable channel and noise models must be taken into account.

In this work we adopt a narrowband PLC (NB-PLC) operating on CENELEC-C band hereafter mentioned as PLCC. BER of this system is evaluated for binary phase shift keying (BPSK) and quadrature phase shift keying (QPSK) modulations in presence of the NB-PLC noise.

\section{RELATED WORK}

Authors in [11] described channel characteristics and a channel model of indoor power lines for local area networks in homes or small offices. Authors in [12] introduced a multipath channel model for NB-PLC in frequency range from $500 \mathrm{kHz}$ to $20 \mathrm{MHz}$. The same model is used in [13] to generate random channels with different length and parameters for in-home PLC. Three approaches for describing channel model in frequency ranges up to $30 \mathrm{MHz}$ are proposed in [14]. Channel characteristics, noise, and standards for NBPLC in 3-500 kHz band investigated in [3]. Authors in [15] proposed a new approach to use multipath model for NBPLC in lower frequency bands. In [16] a NB-PLC channel measured experimentally in frequency range between 150-500 $\mathrm{kHz}$ and proposed to be used in a hybrid wireless/PLC system to improve the reliability of the communication.

Although recently there has been several works on wireless link between SM and IHD [5], to the best knowledge of the authors, there is a limited availability of literature and experimental data addressing PLC-C as a communication link for such application in residential buildings.

The remainder of this work is organized as follows: First main models for PLC channel are provided. In addition, different types of noise which are typical for power line communication are listed. Section IV introduces the system model. In Section V simulation result are presented. Finally, conclusion and future works are drawn in VI.

\section{Channel AND NoIse Models}

Power line channel is a very hostile medium for communication, as it is frequency selective and time-varying. Depending on the type of equipment attached to the channel as well as frequency, location, and time, the properties and parameters of the channel change.

The complexity of the electric network topology and its various connected household appliances induces a frequencyselective propagation channel behavior. In addition, household appliances may generate impulsive noises resulting in some errors on the PLC link or, in worst cases, cause communication or connectivity failures.

Lower frequency ranges are sensitive to interference. The reason is that the impedance is low and noise is high, moreover the bandwidth is proportional to the bit rate, so it is not easy to obtain communication with high speed.

Power line is exposed to background noise, narrow-band interference, and impulsive noise which usually takes place 
at $50 / 60 \mathrm{~Hz}$ [17]. A typical low voltage network between a meter and IHD consists of a distributer cable and branching house connection cables of different characteristic impedance. Each of the transitions at the connections between these cables represent change in the impedance. Impedance mismatches causes reflection. The channel behavior can vary abruptly when the topology changes for example when the connected devices are plugged out.

In the next two subsections, first we introduce different approaches to model the channel and channel model used for our system. Then, different noise and interference of PLC are listed and the mathematical model for modeling noise in our system is presented.

\section{A. Channel Model}

There are two main approaches for modeling the transfer function of the PLC channel:

- Top-down Approach: estimation through measurements.

- Bottom-up Approach: theoretical analysis of Transmission Line Model

In the first method, the propagation model is used in time and frequency domain. The corresponding parameters are obtained from measured channel response. Finally, parameters are adopted to fit the expected values and transfer function is achieved.

In contrast, in bottom-up approach relevant parameters of the channel model are derived theoretically. This method require detailed information about all components in the network.

Most of the top-down channel models are broadband models and only very few of them focus on or cover narrowband or the CENELEC bands (3-500 kHz) [18]. A top-down approach for broadband system is not applicable for narrowband. A bottomup approach is suitable to model the frequency response of the channel in all frequency ranges. This is true while the frequency dependent parameters like impedance and per-unitlength parameters are known in all frequency ranges [18]. Otherwise, measurements are still required to confirm that the model is valid or not [18].

Any signal transmitted on powerlines encounter multipath distortion since the transmitted signal reaches the receiver over more than one path. Each path may have different length, delays and attenuation [19]. Attenuation and reflection take place due to the impedance mismatches in the branching points. However, this phenomenon is less observed below 150 $\mathrm{kHz}$ because the PLC signal wavelength is very long [20].

Zimmermann et.al [12] have proposed an analytic model based on top-down approach to describe the complex transfer function of a powerline network using small set of parameters. Longer paths have higher attenuation, which leads to lower contribution to the overall signal at the receiver. Thus, instead of considering infinite number of paths, multipath scenario can modeled with a number of $\mathrm{N}$ dominant paths. The final sim- plified model for describing the channel model in frequency domain:

$$
\begin{gathered}
H(f)=\sum_{i=1}^{N} g_{i} A(f, d) e^{-j 2 \pi f\left(\frac{d_{i}}{v_{p}}\right)} \\
g_{i}=\prod_{j=1}^{K} \Gamma_{i j} \prod_{k=1}^{M} T_{i k} \\
A(f, d)=e^{-\left(a_{0}+a_{1} \cdot f^{k}\right) \cdot d_{i}}
\end{gathered}
$$

$a_{0}, a_{1}$ : Attenuation parameters

$k$ : Exponent of attenuation factor (typically between 0.5 and 1)

$N$ : Number of dominant paths

$g_{i}$ : Weighting factor which is the product of reflection $\Gamma_{i j}$ and transmission $T_{i j}$ factors

$K$ : Number of reflections occurred in a path between the receiver and transmitter

$M$ : Number of transmissions occurred in a path between the receiver and transmitter

$A(f, d)$ : Attenuation term

$d_{i}$ : Length of the cable

$v_{p}$ : Phase velocity of propagating wave in the cable

This model is for PLC in frequency range between $500 \mathrm{kHz}$ to $20 \mathrm{MHz}$, so for PLC-C we used modified form of multipath model proposed in [15].

\section{B. Noise and Interference Model}

The key parameters which define the quality of the communication channel are: noise, attenuation and multipath effect. In power lines noise is generated by electrical loads attached to the network. The noise is strong, non-white, varying with time and location, and usually non-Gaussian. Moreover, the noise power on these lines is obtained from the summation of various disturbances [21] [22], hence power line applies significant attenuation on the signal and puts a limitation on the distance from the transmitter to the receiver. In addition, the power of noise at lower frequencies is higher, therefore it is more severe for NB-PLC.

Differently from other communication channels, in PLC the noise model cannot be accurately described by simply considering additive white gaussian noise (AWGN). In the case of narrowband PLC systems, the symbol duration tend to be long because of relatively narrow bandwidth (and slowdata rate) thus the impact of periodic impulse noise cannot be ignored. Some of the main types of noise found in typical PLC applications are:

- Colored Background Noise

- Narrow Band Noise

- Impulsive Noise

- Periodic synchronous to AC mains

- Periodic asynchronous to AC mains

- Aperiodic or Asynchronous Impulsive

We adapted the Middleton model which is one of the methods to model the non-Gaussian noise. In this model, 


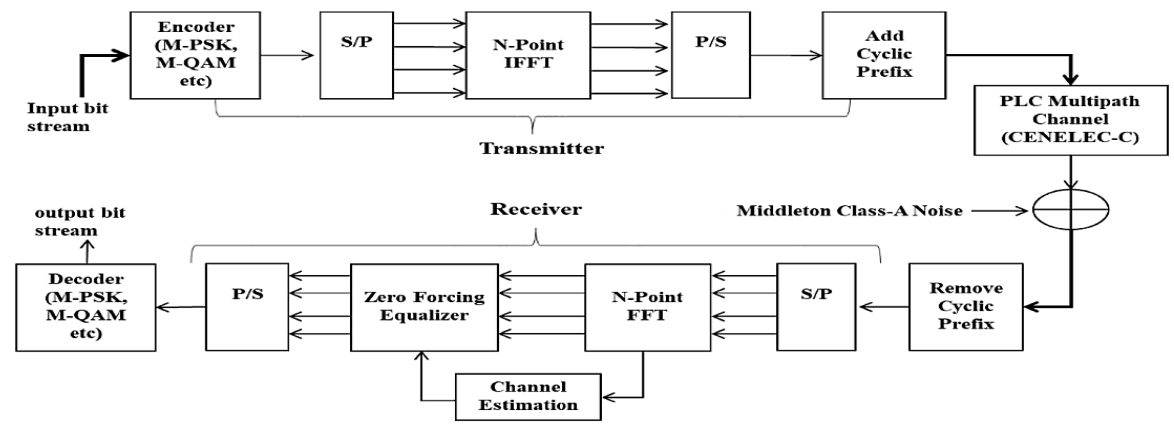

Fig. 2. System Block Diagram for PLC-C Transceiver

probability density function (PDF) of impulsive noise is indicated as superposition of Gaussian functions which have different variances. The benefit of this approach is that by means of few parameters in a simple function, different types of impulsive noise can be modeled. However, this model doesn't cover the time-domain behavior. Middleton model has three classes which are statistical physical models and consist of non-Gaussian components of both natural and man-made noise[23]:

- class A: Narrowband compared to the receiver bandwidth

- class B: Broadband compared to the receiver bandwidth

- class C: a linear sum of class A and class B noise

Class-A is the most used and has been widely accepted to model the effects of impulse noises in communications systems which have negligible transient with respect to the emission's interval[24]. According to this model, PDF of the random variable $n$ representing impulsive noise is expressed as:

$$
P_{n}(n)=\sum_{m=0}^{\infty} p_{m} \frac{1}{\sqrt{2 \pi \sigma_{m}^{2}}} \exp \left(-\frac{n^{2}}{2 \sigma_{m}^{2}}\right)
$$

with

$$
\begin{aligned}
p_{m} & =e^{-A} A^{m} / m ! \\
\sigma_{m}^{2} & =\sigma^{2} \frac{m / A+\Gamma}{1+\Gamma}=\sigma_{i}^{2} \frac{m}{A}+\sigma_{g}^{2} \\
A & =v_{t} T_{s} \\
\Gamma & =\sigma_{g}^{2} / \sigma_{i}^{2}
\end{aligned}
$$

$p_{m}$ : Poisson distribution of impulsive noise

$m$ : Number of origins of impulse noise

$\Gamma$ : Mean power ratio between Gaussian and Impulsive noise elements

$\sigma_{g}^{2}:$ Variance of Gaussian noise

$\sigma_{i}^{2}$ : Variance of Impulsive noise

$A$ : Impulsive index, it is a measure for impulsiveness of the noise.

$v_{t}$ : Average number of impulses in unit time

$T_{s}$ : Mean impulse duration in second

The smaller $A$, the less number of impulses, but the higher the impulses' amplitude. In contrary, the higher $A$, the more pulses with lower amplitude. If $A \rightarrow \infty$ then $P(n)$ tends toward Gaussian PDF. Finally, with low value for both $A$ and $\Gamma$, a very impulsive noise will be resulted.

\section{SYSTEM MODEL}

Figure 2 shows the block diagram of our post-meter PLC-C system which provide meter data directly to the end customer. The metering resolution affects the frequency of information displayed to users, and in a sense the responsiveness of control tasks [25]. This generates traffic in the form of bits which are transmitted by the SM towards the Post-Meter.

The input data stream bits are encoded into continuous signals by the encoder, which is first block of the transmitter. Data encoding can be done by using any of the digital modulation techniques such as M-QAM, M-PSK, or M-FSK. After that $N$ such symbols are transferred by the serial-toparallel converter. These complex parallel data symbols are fed into the IFFT block. After taking N-point IFFT, the last $g$ samples are appended as a cyclic prefix (CP).

The length of CP plays an important role in the performance of OFDM system. If the length of the guard interval is set shorter than the maximum delay of a multipath channel, the tail part of an OFDM symbol affects the head part of the next symbol, resulting in the ISI.These complex parallel data symbols are then modulated by a group of orthogonal subcarriers to form the OFDM signal.

For the channel and noise modeling we refer to Sec III. Channel model is multipath model which introduced by Zimmermann et.al [12]. This model is proposed for the frequency range from $500 \mathrm{kHz}$ to $20 \mathrm{MHz}$, however, CENELEC-C is in the frequency range between $125-140 \mathrm{kHz}$.

Our channel model is based on model proposed in [15] for frequency range between $10-500 \mathrm{kHz}$ for a typical power line cable common for indoor wiring. In our model, we modified the attenuation part of multipath model with the approximation as:

$$
A(f, d)=e^{-\left(a_{0}+a_{1} \cdot f\right) \cdot d_{i}}
$$

This system is associated with the building in the left side of Fig. 1. The physical channel is between M1 and M3, in the basement of the building, and the corresponding IHDs in the first and third floor. Maximum length of the cable from M1 to IHD in first floor is set to $40 \mathrm{~m}$, this length is $80 \mathrm{~m}$ for the third floor. 
TABLE II

SIMULATION PARAMETERS FOR THE CHANNEL MODEL

\begin{tabular}{|l|l|}
\hline$a_{0}$ & $2.44 \times 10^{-4}$ \\
\hline$a_{1}$ & $1.19 \times 10^{-9}$ \\
\hline$\varepsilon_{r}$ & 1.5 \\
\hline $\mathrm{K}$ (Exponent of the attenuation factor) & 1 \\
\hline
\end{tabular}

TABLE III

MULTIPATH PARAMETERS FOR THE SIMULATION OF CASE 1

\begin{tabular}{|c|c|c|c|c|c|}
\hline path & 1 & 2 & 3 & 4 & 5 \\
\hline$d$ (path length) & $42 \mathrm{~m}$ & $46 \mathrm{~m}$ & $56 \mathrm{~m}$ & $86 \mathrm{~m}$ & $96 \mathrm{~m}$ \\
\hline $\begin{array}{l}g \\
\text { (weight factor of } \\
\text { each path) }\end{array}$ & $\overline{0}-6078$ & 0.445 & 0.5765 & 0.0621 & 0.0796 \\
\hline $\begin{array}{l}\tau \\
\text { (delay of each } \\
\text { path) }\end{array}$ & $\begin{array}{l}0.21 \\
\mu s\end{array}$ & $\begin{array}{l}0.23 \\
\mu s\end{array}$ & $\begin{array}{l}0.28 \\
\mu s\end{array}$ & $\begin{array}{l}0.43 \\
\mu s\end{array}$ & $\begin{array}{l}0.48 \\
\mu s\end{array}$ \\
\hline
\end{tabular}

Signal passes through the channel and is subject to noise, we perform the inverse operations at the receiver side to obtain the transmitted signal. After removing the cyclic prefix we perform the FFT in order to move to the frequency domain. Henceforth we estimate and equalize the channel in the frequency domain through the zero forcing equalizer. Lastly, we decode the modulation in order to extract the output bit stream and compare it with the input stream to perform the BER analysis shown in Sec. V.

\section{Simulation Results}

Simulation analysis of the proposed secheme is given in this section. Bit error rate (BER) performance is obtained by Monte Carlo simulation for BPSK and QPSK, respectively. The parameters used for the simulations are reported in the tables II, III and IV.

First of all, the impact of the different length on the performance of OFDM system based on the PLC-Multipath with Middleton class-A Noise has been evaluated. Results for the BPSK case are shown in Fig. 3, where the simulation results, of the different length and also on each length with different number of the multipath are reported in case of transmission over the PLC-multipath with $L=40 \mathrm{~m} L=80$ $\mathrm{m}$ respectively. For each length $L$, we have consider the $\mathrm{nTap}=5$ and $\mathrm{nTap}=12$ multipath.

As it can be observed from the figures, the performance of the system is better when we consider the $L=40 \mathrm{~m}$ with number of the multipath $=5$, as we increase the number of the multipath component we observe the digression in the performance of the system. We also obtained the similar results in the case of the $L=80 \mathrm{~m}$ length. Moreover, similar consideration hold for the QPSK case, for which numerical results are presented in Fig. 4.

From these figures we observe that the impact of multipath contributions weights heavily on the performance of the system, this is expected as the increased number of paths interacts with the impulsive nature of the noise causing greater interference to the signal.
TABLE IV

SIMULATION PARAMETERS FOR THE NOISE MODEL

\begin{tabular}{|l|l|}
\hline $\begin{array}{l}\text { A } \\
\text { (impulsive index) }\end{array}$ & 6 \\
\hline $\begin{array}{l}\Gamma \\
\text { (Gaussian to impulsive noise } \\
\text { power ratio) }\end{array}$ & 0.1 \\
\hline $\begin{array}{l}\text { M } \\
\text { (number of impulsive noise } \\
\text { sources) }\end{array}$ & $\begin{array}{l}2 \text { impulsive source for 5 paths } \\
8 \text { impulsive source for 12 paths }\end{array}$ \\
\hline $\begin{array}{l}\sigma_{i} \\
\text { (Impulsive noise variance) }\end{array}$ & $\frac{\sigma_{g}{ }^{2}}{\Gamma}$ \\
\hline $\begin{array}{l}\sigma_{g} \\
\text { (Gaussian noise variance) }\end{array}$ & $\Gamma \sigma_{i}^{2}$ \\
\hline $\begin{array}{l}\sigma \\
\text { (Total noise variance) }\end{array}$ & $\sqrt{\frac{\frac{S N R_{S} \text { inear }}{N p}}{2}}$ \\
\hline $\begin{array}{l}P_{s} \\
\text { (average signal power) }\end{array}$ & 0.061 \\
\hline SNR & $5-40 \mathrm{~dB}$ \\
\hline
\end{tabular}

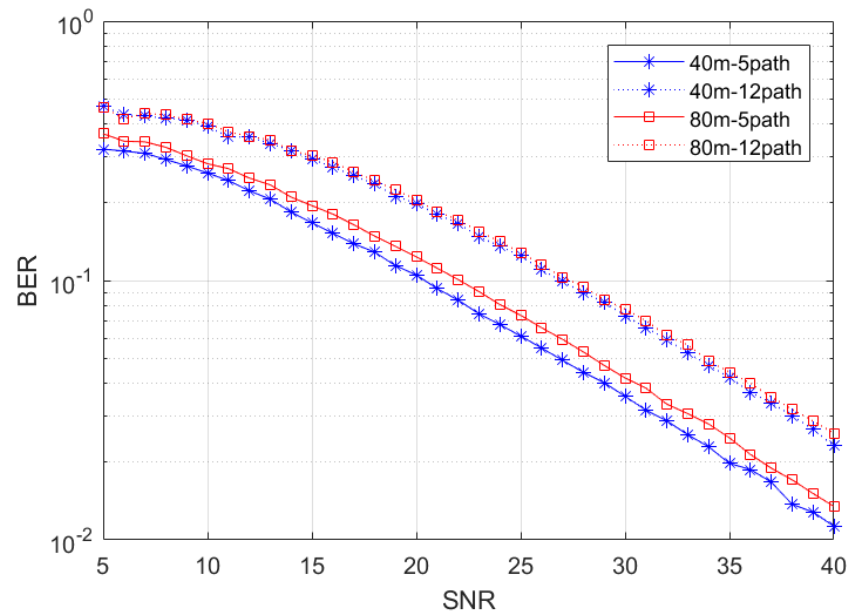

Fig. 3. BER simulations with varying values of SNR using BPSK

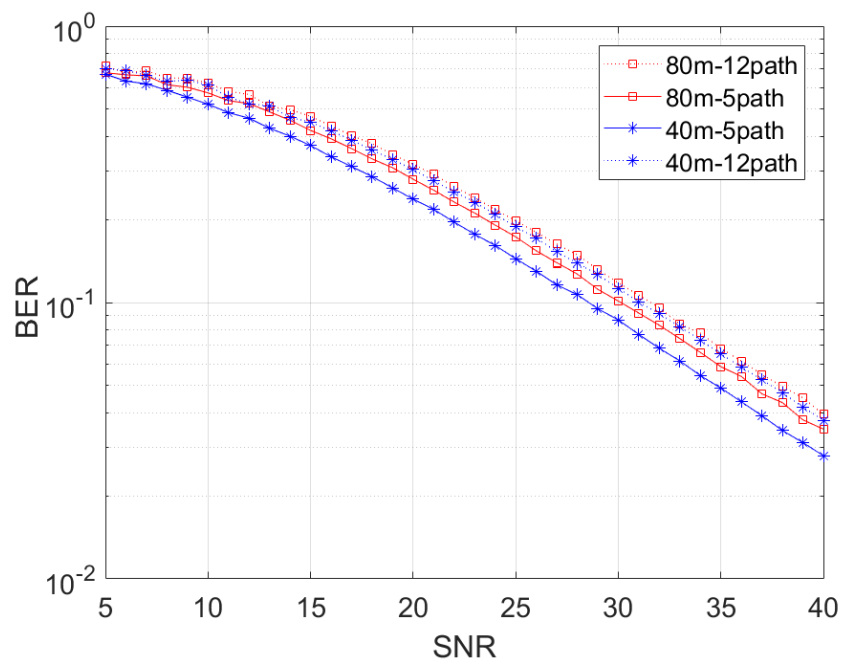

Fig. 4. BER simulations with varying values of SNR using QPSK 


\section{CONCLUSION AND FUTURE WORKS}

In this paper we focused on a narrowband powerline communication (NB-PLC) operating in the CENELEC-C band of $125 \mathrm{kHz}$ to $140 \mathrm{kHz}$. Moreover, We extended the existing higher frequency PLC models in to the case of the lower frequency bands. Here, we also provide the detailed impulsive noise model typical of the applications intended for CENELEC-C band. Performance analysis is done by considering the OFDM system transmission over the PLC mutipath channel (CENELEC-C) with Middleton class-A noise models for different modulation schemes. Here, the performance comparison is done by considering the $40 \mathrm{~m}$ and $80 \mathrm{~m}$ of the path length and, in both cases, we tested the impact of multipath with 5 path and 12 path models. We have observed that an increase in the number of paths impacts heavily on the BER performance. As a future work, we aim to investigate on the effects of different values for parameters of middleton class-A noise on BER. Moreover, other noise models can be used in simulations.

\section{REFERENCES}

[1] D. Schneider, J. Speidel, L. Stadelmeier, D. Schill, and A. Schwager, "Potential of mimo for inhome power line communications," ITGFachtagung, Dortmund, 2009.

[2] L. T. Berger, A. Schwager, and J. J. Escudero-Garzas, "Power line communications for smart grid applications," Journal of Electrical and Computer Engineering, 2013.

[3] M. Nassar, J. Lin, Y. Mortazavi, A. Dabak, I. H. Kim, and B. L. Evans, "Local utility power line communications in the 3-500 khz band: channel impairments, noise, and standards," IEEE signal processing magazine, vol. 29, no. 5, pp. 116-127, 2012.

[4] PL132, Konnex, "Power-line-communication using the cenelec-c-band," DOMOLOGIC Home Automation GmbH,, 2003.

[5] A. Pitì, G. Verticale, C. Rottondi, A. Capone, and L. Lo Schiavo, "The role of smart meters in enabling real-time energy services for households: The italian case," Energies, vol. 10, no. 2, p. 199, 2017.

[6] L. D. Bert, S. D'Alessandro, and A. M. Tonello, "An interconnection approach and performance tests for in-home plc networks," in 2012 IEEE International Symposium on Power Line Communications and Its Applications, March 2012, pp. 182-187.

[7] N. Bizon, N. M. Tabatabaei, F. Blaabjerg, and E. Kurt, Energy Harvesting and Energy Efficiency: Technology, Methods, and Applications. Springer, 2017, vol. 37.

[8] V. Oksman and J. Zhang, "G. hnem: the new itu-t standard on narrowband plc technology," IEEE Communications Magazine, vol. 49, no. 12, 2011.

[9] J. LeClare, A. Niktash, and V. Levi, "An overview, history, and formation of ieee p1901. 2 for narrowband ofdm plc," Maxim Integrated, Application Note, vol. 5676, pp. 1-7.

[10] A. A. Amarsingh, H. A. Latchman, and D. Yang, "Narrowband power line communications: Enabling the smart grid," IEEE Potentials, vol. 33 no. 1, pp. 16-21, Jan 2014.

[11] F. J. Canete, J. A. Cortes, L. Diez, and J. T. Entrambasaguas, "Modeling and evaluation of the indoor power line transmission medium," IEEE Communications Magazine, vol. 41, no. 4, pp. 41-47, April 2003.

[12] M. Zimmermann and K. Dostert, "A multipath model for the powerline channel," IEEE Transactions on communications, vol. 50, no. 4, pp. 553-559, 2002.

[13] A. M. Tonello, F. Versolatto, and B. Béjar, "A top-down random generator for the in-home plc channel," in Global Telecommunications Conference (GLOBECOM 2011), 2011 IEEE. IEEE, 2011, pp. 1-5.

[14] H. Philipps, "Modelling of powerline communication channels," in Conf. IEEE Int. Symp. on Power Line Communications and Its Applications, 1999, pp. 14-21.
[15] H. Gassara, F. Rouissi, and A. Ghazel, "Empirical modeling of the narrowband power line communication channel," in Multimedia Computing and Systems (ICMCS), 2016 5th International Conference on. IEEE, 2016, pp. 570-575.

[16] S. Moaveninejad, A. Saad, and M. Magarini, "Enhancing the performance of winplc smart grid communication with mimo nb-plc," in Environment and Electrical Engineering and 2017 IEEE Industrial and Commercial Power Systems Europe (EEEIC/I\&CPS Europe), 2017 IEEE International Conference on. IEEE, 2017, pp. 1-6.

[17] "Ieee standard for low-frequency (less than $500 \mathrm{khz}$ ) narrowband power line communications for smart grid applications - amendment 1," IEEE Std 1901.2a-2015 (Amendment to IEEE Std 1901.2-2013), pp. 1-28, Oct 2015.

[18] W. Zhu, X. Zhu, E. Lim, and Y. Huang, "State-of-art power line communications channel modelling," Information Technology and Quantitative Management , ITQM 2013, pp. 563-570, 2013.

[19] C. Temaneh-Nyah and P. Chifamba, "An investigation of the effects of impulsive noise and channel distortion in narrow band power line communication," in 2015 Third International Conference on Digital Information, Networking, and Wireless Communications (DINWC), Feb 2015, pp. 16-21.

[20] B. Baraboi et al., "Narrow band power line communication, applications and challenges," Ariane Controls Inc, Québec, 2013.

[21] M. Gotz, M.Rapp, and K.Dostert, "Power line channel characterischar and their effects on communication system design," IEEE Cотти. Mag, pp. 78-86, April 2004.

[22] H. C. Ferreira, L. Lampe, J. Newbury, and T. G.Swart, Power Line Communications: Theory and Applications for Narrowband and Broadband Communications over Power Lines. Wiley, 2010.

[23] D. Middleton, "Non-gaussian noise models in signal processing for telecommunications: new methods an results for class a and class b noise models," Information Theory, IEEE Transactions, vol. 45, no. 4, pp. 1129-1149, May 1999.

[24] - "Statistical-physical models of electromagnetic interference," Electromagnetic Compatibility, IEEE Transactions, pp. 106-127, 1977.

[25] S. D'Alessandro, A. M. Tonello, A. Monacchi, and W. Elmenreich, "Home energy management systems: Design guidelines for the communication infrastructure," in 2014 IEEE International Energy Conference (ENERGYCON), May 2014, pp. 805-812. 